\title{
Digital libraries in research institutes and organizations
}

\author{
S.S. Hiremath \\ Senior Librarian, BLDE (Deemed to be University), Vijayapura, Karnataka, India \\ *Corresponding Author: S.S. Hiremath \\ Email: suvarnah1965@gmail.com
}

\begin{abstract}
In the ear of information and R \& D the new information era where IT makes remarkable progress and internet resources increase rapidly, proper access to information resource is more important than any other things. Especially In users of research Institute organizations demands more sophisticated services from this new digital environment, Such as e-journals, e-books, e-databases and web resources. In order to meet the growing needs for integrated e-resources, a proper digital library with high-quality services is very essential to meet the requirements of the users. This paper goes in to the details of Digital Library Responsibility and its importance in Research Institute and R \& D Organizations.
\end{abstract}

Keywords: Digital Library, Virtual Library, Electronic Library, Library Services, Research Institute, R\&D Organizations.

\section{Introduction}

In this information technology age, it is natural that all kinds of institutions are being affected by technological development, especially users of research institutes and R\&D organizations are more interested in receiving the information services at their work place rather than going to reference desk or to a library each time. Digital library adds value to information resources by organizing them and making them available, additionally it serves distinct sets of stakeholder's communities of frequent, casual and potential users. It plays a vital role to meet the requirements of user community and have been always source of systematized knowledge for various applications. The study is made about what is DL? Why it is necessary? Its purpose, its components and role, importance in meeting the requirements of end user community of Research Institutes and R \& D Organizations.

\section{What are Digital Libraries}

The DL is a library in which collections are stored in digital format and accessible by computers, the digital content may be stored locally or accessed remotely via computer networks. ${ }^{1}$ It also refers to a system or set of applications whose function is chiefly to extend electronic access to material available in a conventional library to remote users.

D L's are complete and advanced forms of information systems that can be endowed with a multiplicity of various functions and features, sometimes these are called as electronic infrastructures and information highways etc, but library is not the same thing as one called it an Infrastructure. ${ }^{2}$

\section{Some Definitions}

Digital libraries (DLs) are computerized, online information systems which organize and store information and knowledge, to view, retrieve and (re)use at any time and at any place with digitized content and computerized services (Arms). ${ }^{3}$

DL has the same knack as were present before, In addition to that there is the potential to eliminate scarcity, reduce staff and physical plant overhead, have content available at all times and from anywhere in the world, have powerful near-instant searching.(Lesk and Michael). ${ }^{4}$
The Information Infrastructure Technology and Applications (IITA) Working Group consider 'digital libraries as systems providing users with coherent access to a very large, organized repository of information and knowledge' (Lynch \& Garcia-Molina). ${ }^{5}$

\section{Why Digital Libraries:}

Some of the factors that necessitate design and development of digital libraries are as fallows. ${ }^{6}$

1. Digital resources have come to be regarded as an increasingly important resource type, this is a direct result of the web being used extensively for scholarly publishing as well as for publishing resources of value in commerce and government. D L serves as portals for the huge reservoirs of information.

2. D L have role in preservation/ archiving information, it presents opportunities for long term preservation of resources.

3. The rising costs of acquisition and subscription to journals have forced librarians to look for other way to make information available.

\section{Characteristics of Digital Libraries:}

The some of the important characteristics of DL are as follows. ${ }^{7}$

1. Whoever wants to use library must show permission to do so

2. Different patterns are permitted different actions and to see different parts of each collections

3. To find specific information, each user must understand the catalogue structure

4. The catalogue may describe items not actually held as part of the collection at hand

5. The catalogue and collected items are used differently and not necessarily housed in the same place.

6. Documents are catalogued with text descriptors and also with conventional properties such as author names.

7. Documents contain cross references to other documents.

8. Documents identifiers are different from document names. 
9. Translations of a document may express essentially the same information eg: version of classic literature in different languages.

10. Each stored item is valuable often with part of its residual value assigned by its authors originally or from others.

11. Part of the value provided by a library is the provenance information it hold for each item.

\section{Components of Digital Library:}

Digital Libraries requires well established and proven information technologies by accessing the databases or servers through networks, the fallowing components are very essentials to create digital library. ${ }^{8}$

\section{Hardware Requirement:}

The major requirements of the DL as indicated below

1. Computer Servers

2. LAN and WAN networks

3. Scanners

4. 24 Hours internet connectivity

5. Storage media: high power hard disk and CD's

6. Digital Camera

7. High power UPS

8. Converters

9. Multimedia Interface

\section{Software Requirement}

The software requirements of D L are indicted below

1. Linux Operating System or windows Operating system

2. Digital Library software like Greenstone, D-space etc

3. Editing Software

Human Ware: The skills required for D L are

1. Management Skills

2. Technical Skills

3. Subject Skills

Objectives of the Digital Libraries: Lynch in 2005 in his article stated that the objectives of digital libraries are as follows. ${ }^{9}$

1. To collect, store, organize and access information in digital form via communication channels

2. To provide personalized and retrospective services in efficient way

3. To have large digitized databases

4. To save time of library staff by avoiding routine jobs

5. To provide coherent view of all information with in a library in any format

6. To serve widely dispersed communities throughout the network

7. To minimize massive storage and space problem of large libraries

8. To reduce cost involved in various library activities

\section{Function of Digital Library:}

The main functions of D L are listed below. ${ }^{10}$

1. Making available various types of Knowledge resources
2. Providing resource discovery mechanisms that allow users to identify revelent or required resources and their locations

3. Providing mechanisms for delivery of specific resources to the user, delivery includes both obtaining a resource when it is not already available in the library and passing it on to the users in a suitable way.

4. Repository service, where digital objects can be deposited and stored \& to which the digital library provide access

5. A naming service, which ensures that each digital object has a globally unique name and that can be resolved in to at least one physical location

6. An index service which enables sets of digital objects to be described and queries to be solved in to result sets containing unique names

7. A collection service which performs selection against defined criteria tailored and specialized catalogues or other resource discovery aids and administrative process

Digital conversion process: Digital conversion process includes.

Document

Data Processing

: Data Capture

Indexing and Periodicals : Retrieval and Display

\section{Document}

It includes text, bibliographic or full text, photographs, diagrams, chart, maps and colour images, etc they exists either in print or non print form.

\section{Data Capture}

It includes manual data entry (word processing) optical character recognition (OCR) or imaging using scanners.

\section{Data Processing}

The text in the convertible document may require conversion of diacritics or special characters, images may need enhancement, amplification or compression, in many cases a simple conversion from print to digital is not enough.

\section{Storage}

The digital information needs to be recorded in proper digital storage medium which may be hard disk, magnetic tape, optical CD-ROM or Networks with workstation to access.

\section{Indexing and Processing}

Digitized document need to be processed using standards protocols and indexing systems, classification using library systems also held much promise, metadata application should be a major component of the digital information processing.

\section{Retrieval / display}

It is the process through which an array of technologies for browsing displaying and applying packages that ultimately helps in access. 


\section{Role and Importance in Research Institutes and R\& D organizations}

The major roles of DL in Research Institute and R\&D organizations are

1. It helps in Resource Sharing

2. It saves the time and energy of the researchers by providing instant access to information resources

3. It helps in Interlibrary Loan (I LL)

4. Helps to reach information of their users at faster rate through on-time communication

5. It minimizes the duplication of new invention

6. It Helps the libraries to get recent publications from the publishers

7. Researchers, Information scientist will get information with minimum time

8. It helps to get Bibliographic information, Retrospective search, Union catalogue, Abstracting and Indexing of periodicals

9. Online library catalogue through internet gives access to bibliographic records of millions of books and details of holdings of academic \& research Libraries, e-journals and news letters

10. E-publications provide aids for connectivity audio visualization customizability, creation and revision of documents, interactivity and rapid information retrieval

11. E-publication may help in overcoming the restrictions on the length of the paper imposed by many scholarly journals

12. The E-publications data can be maintained up to date so that researcher will be able to access the latest version of the publications this enables on demand publishing and allows retrospective searching and SDI

\section{Conclusion}

The paper discussed the role of DL and its function, tools, technologies used for information dissemination to their users, Here everything is found in digital form, it has transformed the methods of creating, processing, archiving and disseminating information and knowledge resources to the genuine users of RI and R\& D organization, this new dimension has changed the scope of libraries and their users to a maximum extent.

\section{Conflict of Interest}

The authors declare that there are no conflicts of interest in this paper.

\section{Source of Funding}

None.

\section{References}

1. Definition of Digital Library available at http://en.wikipedia.org/wiki/digital_library.

2. Nunberg, Geoffrey. (1993). The place of books in the age of electronic reproduction.

3. Arms W. (2000). Digital libraries. Cambridge, MA: MIT Press, p2.

4. Lesk and Michael. (1997). Practical digital libraries: books, bytes and bucks. California, Morgan Kauffmann Publishers, pp1-2.

5. Lynch \& Garcia, Molina. (2000). Interoperability, scaling, and the digital libraries research agenda: A report on the May 1819, 1995, IITA digital libraries workshop.

6. Howard, Block \& Carla, Hesse. Edited 'Representations special issue: future libraries' No 42, CA, Berkeley, pp 13-17.

7. Nazeer, Badhusha K. Digital library architecture, New delhi: Ane books India. pp 2008;41-53.

8. Domain, David. (1998). "The E-book: pipe dream or potential disaster" American Libraries, 30(2), pp18-21.

9. "Preservation of Library resources in digital environment" University News: A Weekly. J Higher Educ. 2006;44(11):13-9.

10. Lynch, Clifford. (2005). "Where do we go from here? The next decade for digital Libraries” D-Lib Magzine, 2005;11(7/8).

How to cite: Hiremath SS. Digital libraries in research institutes and organizations. IP Indian J Libr Sci Inf Technol. 2021;6(1):30-2. 